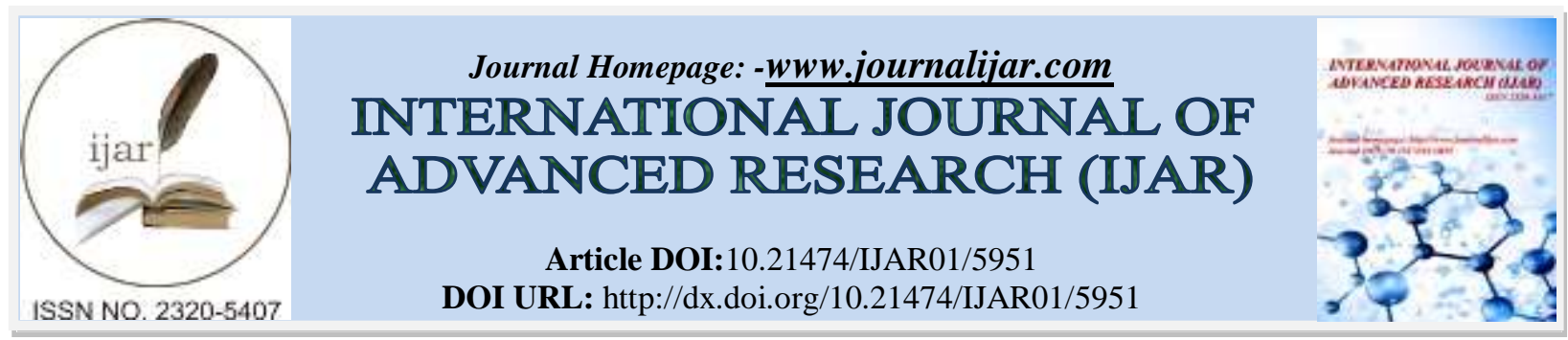

RESEARCH ARTICLE

\title{
PLASMA HOMOCYSTEINE LEVELS AND SERUM LIPID PROFILE IN PATIENTS WITH RETINAL VENOUS OCCLUSIVE DISORDERS: AN OBSERVATIONAL STUDY.
}

Dr.P.Manjula MS, DO ${ }^{1}$ andDr. Ch. Ramakrishna MBBS $^{2}$.

1. Assistant Professor, Ophthalmology, Andhra Medical College, Visakhapatnam.

2. Junior Resident, Ophthalmology, Andhra Medical College, Visakhapatnam.

\section{Manuscript Info}

Manuscript History

Received: 02 October 2017

Final Accepted: 04 November 2017

Published: December 2017

Key words:-

Plasma Homocysteine Levels, Serum

Lipid Profile, Retinal Venous

Occlusions, CRVO, HRVO, BRVO.

\section{Abstract}

Aim: To observe the levels of Plasma Homocysteine and Serum Lipids in patients diagnosed with Retinal Vein Occlusions.

Methods: 25 patients diagnosed with Retinal Venous Occlusion (CRVO, BRVO, HRVO) with age of less than or equal to 50 years which constituted 11 males and 14 females with mean age of 44.52 years were investigated for Plasma Homocysteine Levels and Serum Lipid Profile.

Results: Of the 25 patients of retinal venous Occlusion, levels of Plasma Homocysteineof more than $12 \mu \mathrm{mol} / \mathrm{L}$ were observed in 22 patients accounting for $88 \%$ of the cases. Total Cholesterol levels were elevated above normal in 13 patients accounting for $52 \%$ of the cases. Serum HDL cholesterol levels were reduced below normal in 6 patients ( $24 \%$ of cases). Serum LDL cholesterol levels were elevated above normal in 17 patients (68\% of cases). Serum VLDL Cholesterol levels were elevated above normal in 12 patients (48\% of cases). Serum Triglyceride levels were elevated in 5 patients (20\% of cases).

Conclusion: In our study Homocysteine levels were elevated in 22 patients (88\% of cases) with Retinal Venous Occlusion Disorder and Dyslipidemia (Hypercholesterolemia) was observed in 13 patients accounting for $52 \%$ of cases. Hence detection and management of systemic risk factors helps in reducing the risk of occurrence of future Ocular as well as Systemic vaso-occlusive events.

Copy Right, IJAR, 2017,. All rights reserved.

\section{Introduction:-}

Many systemic risk factors have been associated with retinal vein occlusion (RVO) with the most common associations being older age, hypertension, hyperlipidemia and atherosclerotic cardiovascular disease; however, their direct relationship to pathogenesis remains speculative.

Completely normal medical and laboratory evaluation results are found in about a quarter of patients. ${ }^{1}$ The global impact of RVOs is significant with an estimated 16.4 million adults affected worldwide. ${ }^{2}$ Large, population based studies have shown that the incidence of RVO over a $10-15$ year period is $1.6-2.3 \%$, with a 15 year cumulative incidence of BRVO of $1.8 \%$ and CRVO of $0.5 \% .^{3}$ The estimated prevalence of BRVO and CRVO is 4.42 and 0.8 per 1000 persons, respectively; prevalence increases with age and does not differ by gender. ${ }^{2}$ The presence of openangle glaucoma is a risk factor for the development of both BRVO and CRVO, with an odds ratio of 2.53 and 9.28 
respectively. ${ }^{3}$ Bilaterality is uncommon in both BRVO and CRVO, with a coincident second RVO occurring in $6.3 \%$ eyes in the 15 year Beaver Dam Eye Study cohort ${ }^{3}$ and in $6.4 \%$ of eyes after 5 years in the Blue Mountains Eye Study cohort. ${ }^{4}$ The risk of any vascular occlusion in the fellow eye has also been estimated to be $0.9 \%$ per year. ${ }^{5}$

The various Local and Systemic risk factors identified for retinal venous Occlusive Diseases are:

- Age is the most important factor; over 50\% of cases occur in patients older than 65.

- Hypertension is present in two-thirds or more of RVO patients over the age of 50 years and in $25 \%$ of younger patients. It is most prevalent in patients with BRVO.

- Hyperlipidaemiais present in one-third or more of patients, irrespective of age.

- Diabetes mellitus is present in up to $15 \%$ of patients over 50 years of age overall. It is more prevalent in Asian and black patients, but uncommon in younger patients.

- Glaucoma and probably ocular hypertension are associated with a higher risk of CRVO and possibly BRVO.

- Oral contraceptive pill. In younger females the contraceptive pill is the most common underlying association, and probably should not be taken following RVO.

- Smoking. Current smoking may be associated with an increased incidence of RVO, though studies have shown inconsistent results.

- Uncommon. Dehydration, myeloproliferative disorders (e.g. myeloma, polycythemia), thrombophilia (e.g. Hyperhomocystinemia, antiphospholipid antibody syndrome, factor V Leiden mutation), inflammatory disease associated with occlusive peri phlebitis (e.g. Behçet syndrome, sarcoidosis, Wegener granulomatosis), orbital disease and chronic renal failure. ${ }^{6}$

The detection and management of associated systemic disease is aimed principally at reducing the risk of future vascular occlusive events, both ocular and systemic. ${ }^{6}$

Various Investigations recommended in patients of Retinal Venous Occlusive diseases are:

All patients

- Blood pressure (BP).

- Erythrocyte sedimentation rate (ESR) or plasma viscosity(PV).

- Full blood count (FBC).

- Random blood glucose. Further assessment for diabetes if indicated.

- Random total and high-density lipoprotein (HDL) cholesterol. Additional lipid testing may be considered.

- Plasma protein electrophoresis. To detect dysproteinemias such as multiple myeloma.

- Other tests. Some authorities advocate routine investigation for systemic end-organ damage related to the cardiovascular risk factors commonly found in patients with RVO. This is intended to help the prevention of further non-ocular damage, as well as facilitating systemic management to reduce the risk of recurrent ocular venous occlusion. Research is conflicting, some studies suggesting that cardio- and cerebrovascular mortality is not elevated above that of the general population in patients with RVO and others finding the converse.

- Urea, electrolytes and creatinine to detect renal disease associated with hypertension; chronic renal failure is also a rare cause of RVO.

$\circ$ Thyroid function testing. There is a higher prevalence of thyroid disease in RVO patients.

$\circ$ Electrocardiography (ECG). Left ventricular hypertrophy is associated with hypertension. ${ }^{6}$

Selected patients according to clinical indication:

These tests might be considered in patients under the age of 50, in bilateral RVO, patients with previous thromboses or a family history of thrombosis, and some patients in whom investigation

for the common associations is negative. Evidence of a causative link for many of these is limited.

- Chest X-ray. Sarcoidosis, tuberculosis, left ventricular hypertrophy in hypertension.

- C-reactive protein (CRP). Sensitive indicator of inflammation.

- Plasma homocysteine level. To exclude Hyperhomocystinemia, for which there is reasonable evidence of an increased RVO risk.

- 'Thrombophilia screen'.By convention this refers to heritable thrombophilias; tests might typically include thrombin time, prothrombin time and activated partial thromboplastin time, antithrombin functional assay, protein C, protein S, activated protein C resistance, factor V Leiden mutation, prothrombin G20210A mutation, lupus anticoagulant and anticardiolipin antibody $(\operatorname{IgG}$ and $\operatorname{IgM})$; the last may be the most important of these. 
- Autoantibodies. Rheumatoid factor, antinuclear antibody (ANA), anti-DNA antibody, antineutrophil cytoplasmic antibody (ANCA).

- Serum angiotensin-converting enzyme (ACE). Sarcoidosis.

- Treponemal serology

- Carotid duplex imaging to exclude mimicking ocular ischemic syndrome ${ }^{6}$

Hyperhomocystinemia and Dyslipidemia were considered as independent risk factors in retinal venous occlusion disorders and hence this study was taken up to find the correlation between Plasma homocysteine levels with serum lipid levels in patients of Retinal Venous Occlusive Disorders.

\section{Materials and Methods:- Inclusion Criteria:-}

Patients attending to the Retina Clinic at Government Regional Eye Hospital, Andhra Medical College, Visakhapatnam diagnosed with Retinal Venous Occlusions between the period of August and November 2017 with age less than 50 years were recruited for the study. Informed consent was obtained from the subject prior to the study.

Methodology:-

A total of 25 patients of retinal vein occlusion were included in the study as per the inclusion criteria. Identification details of the patient like name, age, sex and address were noted. A detailed history regarding the ocular illness was recorded from all the participants of the study. All the patients underwent complete systemic examination in which recording of the vital data was done which included Pulse rate, Blood Pressure and auscultation of the cardiovascular and the Respiratory Systems.

Local examination of both the eyes was done which included:

1.Visual acuity both uncorrected and best corrected.

2.Anterior segment examination by slit lamp.

3.Pupillary reaction was noted to find the RAPD.

4.Fundus examination was done by Indirect Ophthalmoscopy and also with 78D lens with slit Lamp Bio-microscopy 5.IOP measurement was done by Schiotz Tonometer.

Investigations were done which Included:

1.Complete Blood Count(CBC),

2.Post Prandial Blood Sugar(PPBS)

3.Serum Lipid Profile

4.SerumCreatinine Levels

5.PlasmaHomocysteine Levels

Hyperhomocystinemia was defined as Plasma Homocysteine Levels $>12 \mu \mathrm{mol} / \mathrm{L}$

Hyperlipidemia was defined as:

S. cholesterol > $200 \mathrm{mg} / \mathrm{dl}$, S. triglycerides $>200 \mathrm{mg} / \mathrm{dl}$, HDL cholesterol <40 mg/dl, LDL cholesterol > $100 \mathrm{mg} / \mathrm{dl}$, VLDL cholesterol $>30 \mathrm{mg} / \mathrm{dl}$.

\section{Statistical Analysis:-}

Data is analyzed with the help of descriptive statistics.

\section{Results:-}

In our study out of the 25 patients diagnosed with retinal venous occlusions there were 11 males (44\%) and 14 females (56\%) in the study(Fig-1). The minimum age of the participants in the study was 25 years and the maximum age was 50 years, with a mean age of 43.72 years. There were 8 cases of CRVO (32\%), 1 case of HRVO (4\%) and 16 cases of BRVO (64\%)(Fig-2). Of the 25 patients of retinal venous Occlusion, levels of Plasma Homocysteine of more than $12 \mu \mathrm{mol} / \mathrm{L}$ were observed in 22 patients accounting for $88 \%$ of the cases(Fig-3). Total Cholesterol levels were elevated above normal in 13 patients accounting for $52 \%$ of the cases. Serum HDL cholesterol levels were reduced in 6 patients (24\% of cases). Serum LDL cholesterol levels were elevated in 17 patients (68\% of cases). 
Serum VLDL Cholesterol levels were elevated in 12 patients (48\% of cases). Serum Triglyceride levels were elevated in 5 patients (20\% of cases) (Fig-4).

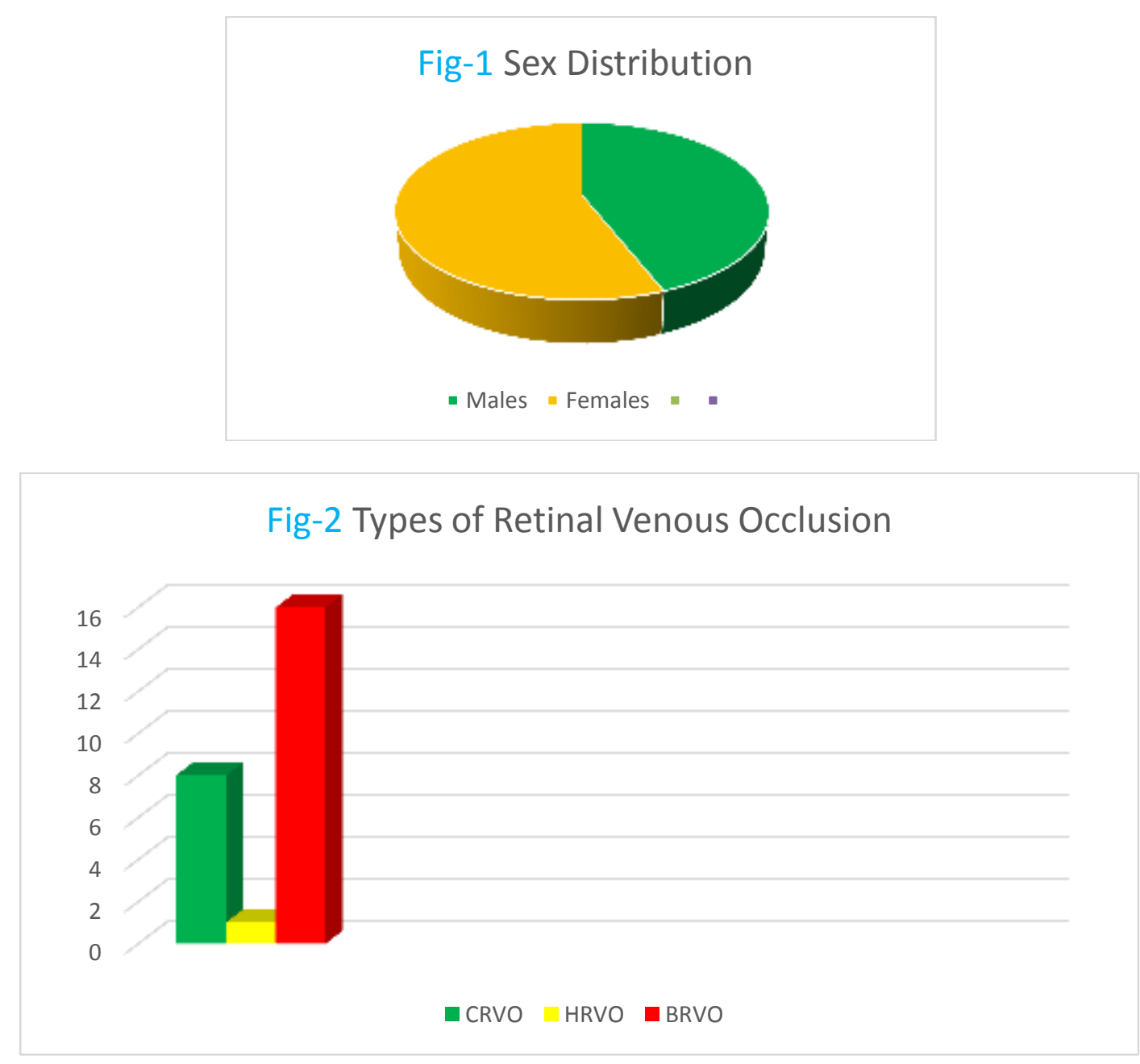

Fig-3 Serum Homocysteine Levels

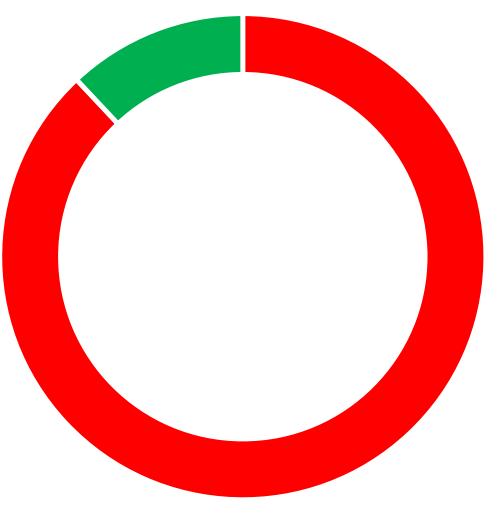

- Serum Homocysteine Abnormal

- Serum Homocysteine Normal 


\section{Fig-4 Serum Lipid Profile}

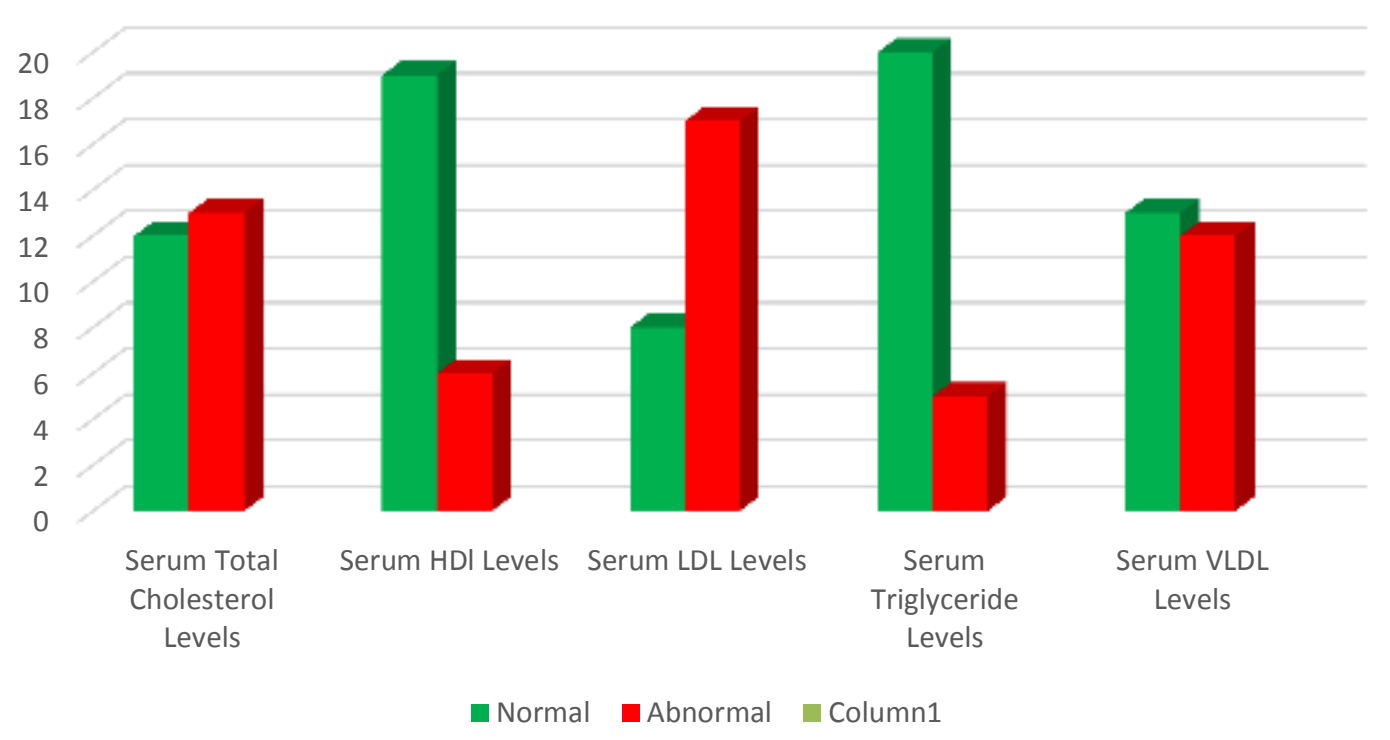

\section{Discussion:-}

Various local and systemic risk factors have been found to be associated with occurrence of Retinal Venous Occlusions. Hyperhomocystinemia and Dyslipidemia were the two systemic risk factors we included in the study. A total of 25 patients diagnosed with retinal venous occlusions below the age of 50 years were included in the study so as to emphasize the role of ruling out dyslipidemia and Hyperhomocystinemia in the younger age group people, as its prompt management might help in reducing the occurrence of future ocular as well as systemic Vaso-occlusive events as the life expectancy is more for this age group compared to the elderly age group people.

This study shows that in population below the age of 50 years with Retinal venous occlusion disorder there is a necessity to screen for Hyperhomocystinemia and dyslipidemia as more than $50 \%$ of the patients in the study have shown the presence of these abnormalities. Elevated Homocysteine levels of more than $12 \mu$ mol/L were observed in 22 cases out of 25 accounting for $88 \%$ of the cases (Fig3). Dyslipidemia (Hypercholesterolemia) was seen in 13 patients accounting for $52 \%$ of cases(Fig-4).

In a study conducted at Government Medical College Kozhikode, Kerala, India, they concluded that Detailed evaluation for systemic risk factors should be made mandatory in all cases of retinal vein occlusion. Many of the patients belonged to older age group and dyslipidemia was the major single modifiable risk factor among these. It should be kept in mind that in younger population, conventional metabolic risk factors may not be evident in the early stages and hence less common factors like high serum levels of homocysteine should be looked for and prompt treatment initiated at the earliest. Strict follow up of these patients for evaluation of any systemic diseases in the future should also be done. ${ }^{7}$

In another study titled "Homocysteine in retinal Vascular Occlusions" it was concluded that: The role of Homocysteine in CRVO is complex. Observational studies have shown that CRVO in general population is associated with raised plasma Homocysteine concentration. ${ }^{8}$

Another population based study by Chua $\mathrm{B}^{1}$, Kifley A, Wong TY, Mitchell P, showed that elevated serum homocysteine is associated with the presence of RVO, independent of other risk factors. ${ }^{9}$

In another study conducted by Rajini sharma1 and MohdAyaz Bhat2 at Department of Ophthalmology, Government Medical College, Jammu titled "Risk Factors in retinal Vein Occlusion", they concluded that Hypertension and hyperlipidemias were strongly associated with RVO (Retinal venous Occlusion). ${ }^{10}$ 
In a study conducted by Kapil Deb Lahiri1, Arunava Kundu2, Joya Ghosh1, Mriganka Baruah1, Champakali Biswas2, Amitava Das2,Nazneen Nazm2 titled "A Study Of Correlation Of Plasma Homocysteine With Serum Lipid Profile In Retinal Vein Occlusion", they found that significant negative correlation was found between homocysteine and HDL cholesterol in RVO patients $(r=-0.273, \mathrm{P}<0.029)$. They concluded that Patients with low HDL cholesterol should be screened for Hyperhomocystinemia as association of low HDL cholesterol and Hyperhomocystinemia might have a synergistic effect on the retinal circulation. ${ }^{11}$

\section{Conclusion:-}

Hyperhomocystinemia and Dyslipidemia have been observed in majority of the cases of Retinal Venous Occlusions in age group of less than 50 years in our study. Hence detection and management of these systemic risk factors helps in reducing the risk of occurrence of future Ocular as well as Systemic vaso-occlusive events.

\section{References:-}

1. Quinlan PM, Elman MJ, Bhatt AK, et al. The natural course of central retinal vein occlusion. Am J Ophthalmol 1990;110(2):118-23.

2. Rogers S, McIntosh RL, Cheung N, et al. The prevalence of retinal vein occlusion: pooled data from population studies from the United States, Europe, Asia, and Australia. Ophthalmology 2010;117(2):313-19.

3. Klein R, Moss SE, Meuer SM, et al. The 15-year cumulative incidence of retinal vein occlusion: the Beaver Dam Eye Study. Arch Ophthalmol 2008;126(4):513-18.

4. Cugati S, Wang JJ, Rochtchina E, et al. Ten-year incidence of retinal vein occlusion in an older population: the Blue Mountains Eye Study. Arch Ophthalmol 2006;124(5):726-32.

5. Natural history and clinical management of central retinal vein occlusion. The Central Vein Occlusion Study Group. Arch Ophthalmol 1997;115(4):486-91.

6. Kanski's Clinical Ophthalmology, $8^{\text {th }}$ Edition, Retinal Vascular Disease, Chapter 13:538-539.

7. Arun Kumar Ayoor et al, Retinal Vein Occlusion -Risk Factors in Younger Patients JMSCR Volume 05 Issue 10 October 2017;29256-29263.

8. Dr. Tirupati Nath1, Dr.Rachit Agrawal2, Dr.Gunjan Prakash3, Homocysteine in Retinal Vascular OcclusionsInternational Journal of Science and Research (IJSR),Volume 5 Issue 1, January 2016;1949-1951.

9. Chua $\mathrm{B}^{1}$, Kifley A, Wong TY, Mitchell P.Homocysteine and retinal vein occlusion: a population-based study. Am J Ophthalmol. 2005 Jan;139(1):181-2.

10. Rajini Sharma1, MohdAyaz Bhat2, Risk Factors in Retinal Vein Occlusion.

11. Kapil Deb Lahiril, Arunava Kundu2, Joya Ghosh1, Mriganka Baruah1, Champakali Biswas2, Amitava Das2,Nazneen Nazm2. A Study Of Correlation Of Plasma Homocysteine With Serum Lipid Profile In Retinal Vein Occlusion. 